ORIGINAL RESEARCH

\title{
Pathophysiologic Determination of Frostbite Under High Altitude Environment Simulation in Sprague-Dawley Rats
}

\author{
Jie Hu, MD; Hua Li, MD; Xiliang Geng, MD; Lin Jiao, MD; Hongping Song, MD; Lin Lou, MD; Mingke Jiao, MD \\ From the Department of Echocardiography, Affiliated Traditional Chinese Medicine Hospital, Xinjiang Medical University, Urumqi, China (Drs \\ $\mathrm{Hu}$ and Li); the Department of Medical Engineering, Urumchi General Hospital of Lanzhou Military Region, Urumchi, China (Drs Geng, Jiao, \\ Lou, and Jiao); and the Department of Ultrasound, Xijing Hospital, The Fourth Military Medical University, Xi'an, China (Dr Song).
}

\begin{abstract}
Objectives.-Pathophysiologic changes of frostbite have been postulated but rarely understood, especially the changes caused by chilly high altitude environment. Therefore, we investigated the pathophysiologic changes of high altitude frostbite (HAF) caused by cold and hypoxia.

Methods.-Forty Sprague-Dawley rats were randomly divided into 5 equal groups, namely, control, superficial HAF (S-HAF), partial-thickness HAF (PT-HAF), full-thickness HAF (FT-HAF), and partialthickness normal frostbite (PT-NF) groups. The S-HAF, PT-HAF, and FT-HAF groups were fed under hypobaric hypoxic conditions simulating an altitude of $5000 \mathrm{~m}$. Then, the PT-NF, S-HAF, PT-HAF, and FT-HAF models were constructed by controlling the duration of the direct freezing by liquid nitrogen. Animal vital signs were measured after the operation, and histopathologic changes were observed after routine hematoxylin and eosin staining. In addition, the microcirculation of frostbite tissues was monitored and compared by contrast ultrasonography during wound healing.

Results.-The S-HAF, PT-HAF, and FT-HAF groups showed significant differences in the microcirculatory and histopathologic changes in the various tissue layers $(P<.05)$. In addition, combined cold and hypoxia caused more damage to frostbite tissue than pure cold. The circulation recovery of HAF models was significantly slower relative to NF models $(P<.05)$.

Conclusions.-A safe and reproducible HAF model was proposed. More important, pathophysiologic determination of HAF provided the foundation and potential for developing novel and effective frostbite therapies.
\end{abstract}

Key words: pathophysiology, high altitude frostbite, high altitude environment, microcirculation, contrast ultrasonography

\section{Introduction}

Frostbite is the acute freezing of tissues caused by exposure to harsh, low-temperature environments. ${ }^{1,2}$ Different populations suffer from severe cold damage., Frostbite is the most common cold injury of outdoor workers undergoing activities in high altitude and frigid regions., 5 Moreover, frostbite injuries usually occur among cold-storage depots as a result of accidental misuse of medical cold devices. ${ }^{7}$ Soldier deaths caused by

Corresponding author: Mingke Jiao, MD, Department of Medical Engineering, Urumchi General Hospital of Lanzhou Military Region, 359 Youhao Road, Urumchi, China 830000 (e-mail: 82161659@ qq.com).

Submitted for publication December 2015.

Accepted for publication March 2016. frostbite are common at subzero environments during wartime. ${ }^{4}$ Frostbite causes pain, productivity loss, high expenditures, and potential for permanent disability. 8,9 However, few studies have indicated allegedly successful treatment of cold injuries in small experiments or case reports. $^{10-16}$ Screening of new and effective therapies using convenient nonhuman animal experiments is essential. Thus, some of the current studies have observed the pathophysiologic changes of frostbite models and showed important developments. ${ }^{10,17-19}$ However, the pathophysiologic determination of frostbite was incomplete for the design of frostbite models. Some frostbite models were restricted by the applied techniques to a designated part of animals, such as ear and leg. ${ }^{20-22}$ In addition, the depth of frostbite was also limited to some certain tissue layers for different reasons. ${ }^{17,18}$ The most important factor was the 
fact that current frostbite pathophysiologic models were formulated by only considering the direct injury of cold, but injury from combined cold and tissue hypoxia was ignored. ${ }^{17-22}$ However, frostbite frequently occurs among high altitude climbers, which results not only from severe cold but also from tissue hypoxia. ${ }^{5,23,24}$ Hypoxia and cold cause increases in blood packed-cell volume, viscosity, and small vessel blockage, which are the main contributors to frostbite injuries. ${ }^{25,26}$ Therefore, more suitable frostbite models and pathophysiologic determination should be implemented to elucidate clearly and completely high altitude frostbite (HAF) pathophysiology and obtain suitable reference for clinical practical application.

A novel HAF model was developed in the present study to determine the pathophysiology of frostbite. In particular, HAF in this paper means the frostbite was caused by cold at a simulated altitude equivalent to approximately $5000 \mathrm{~m}$ above sea level. The HAF models were completed by causing superficial (S-HAF), partialthickness (PT-HAF), and full-thickness (FT-HAF) frostbite injury in the dorsum of Sprague-Dawley rats under cold and hypoxic environment simulation. We observed and determined the pathophysiology of accidental HAF in this paper. Vital signs, as well as microcirculatory and histologic changes, were observed systematically and compared using contrast ultrasonography and light microscopy.

\section{Material and Methods}

\section{ANIMALS}

A total of 40 Sprague-Dawley rats (3-month-old males weighing $275 \pm 10 \mathrm{~g}$ ) were obtained from the Animal Center of the Urumqi General Hospital of Lanzhou Military Region. All uses of animals and experimental procedures followed the guiding principles of the Institutional Animal Ethical Committee, Committee for the Purpose of Control and Supervision of Experiments on Animals, Guide for the Care and Use of Laboratory Animals published by the National Institutes of Health (NIH Publication No. 85-23) and the Urumqi General Hospital Institution Review Board. The rats were allowed to adapt to the hypoxia and low-pressure conditions for 1 week before testing. Animals were housed in a large hypobaric chamber (DYC-3013M, Urumqi General Hospital of Lanzhou Military Region, Urumqi, China) with controlled temperature $\left(23^{\circ} \pm 2^{\circ} \mathrm{C}\right)$, humidity $(50 \% \pm$ $10 \%)$, pressure $(54.1 \pm 1.0 \mathrm{kPa})$, and light-dark cycles (9:00 AM-9:00 PM with lights on). Significantly, $54.1 \pm 1.0$ $\mathrm{kPa}$ is the atmospheric pressure one expects at approximately $5000 \mathrm{~m}$ (17,000 feet) above sea level. The rats received standard chow and clean tap water ad libitum from an automated watering system during the entire experiment.

\section{EXPERIMENTAL DESIGN}

A total of 40 rats were equally divided $(n=8)$ and randomly assigned to 5 groups, as follows: control, S-HAF, PT-HAF, FT-HAF, and PT-normal frostbite (NF) groups. The HAF groups (except the control and the PT-NF groups) were acclimated to a hypoxic and lowpressure environment for 1 week. Blood samples were collected from the tail vein to evaluate the hemorheology and inspected for any disease signs. The rats in the HAF and NF groups were anesthetized by intraperitoneal injection of sodium pentobarbital $(65 \mathrm{mg} / \mathrm{kg}$ body mass). We selected the dorsum to freeze to produce frostbite although different parts of the animals could be frozen to form frostbite in our study. Theoretically, the chill could easily reach different layers because of the thin tissue layers in the dorsum. The dorsum was shaved, with a 35$\mathrm{mm}$ diameter, and the operative site was prepared aseptically before freezing after adequate anesthesia.

The cold-injury equipment used custom-built liquid nitrogen penetration. Liquid nitrogen was stored in an aluminum alloy can with an air sandwich layer. The can was sealed with a resin plug, which consisted of an intake orifice and a gas leak bolt. The container surface was covered with a silicon dioxide aerogel flexible heat insulation film to protect the operator. Air was pumped into the aluminum alloy container by a micropump through plastic pipes to create a pressure gradient, which allowed the liquid nitrogen to penetrate the aluminum alloy connection. A mesh-hole construction and asbestos stopped the container bottom.

The skin of the rat dorsum was made to touch the aluminum alloy mesh upon freezing, which ensured that the liquid nitrogen directly froze the rat tissues. Liquid nitrogen penetration flow was controlled by loosening or tightening the gas leak bolt (Figure 1). The freezing duration was varied to control the frostbite degree.

The freezing duration was limited to 5,15 , and 28 seconds to generate S-HAF, PT-HAF, and FT-HAF, respectively. To compare the HAF group with the NF group, the same process was performed to generate PTNF with the freezing time of 15 seconds. The different effective freezing durations for inducing replicable $\mathrm{HAF}$ models have been studied based on the injury that reached the epidermis, dermis, and muscle layer. Regarding the thickness or depth of the frostbite, the following were observed: S-HAF injury extends to the epidermis; PT-HAF injury extends through the epidermis into the dermis; and FT-HAF injury extends through the dermis into muscle and even bone. The results were confirmed by histologic observation in previous experiments. After surgery, rats were housed individually and fed under normal temperature $\left(23^{\circ} \pm 2^{\circ} \mathrm{C}\right)$ and pressure $(91.5 \pm 0.1 \mathrm{kPa}$, 


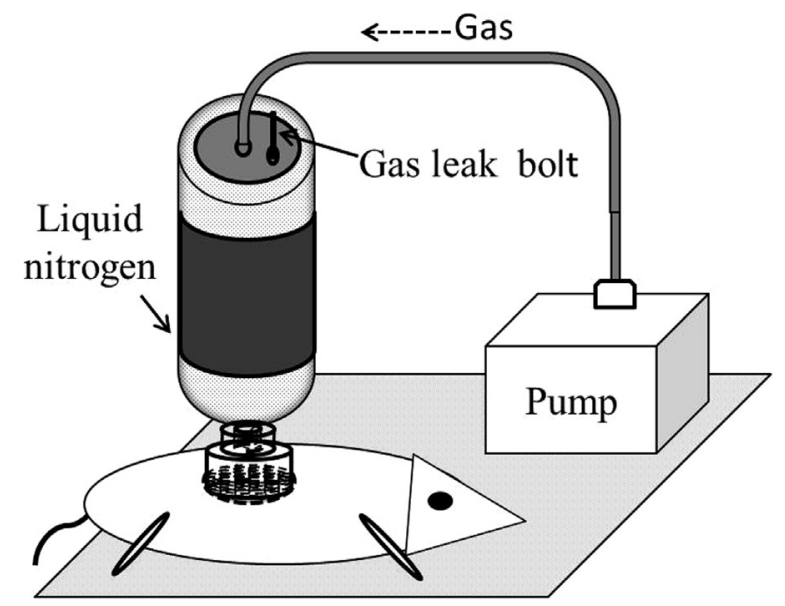

Figure 1. Liquid nitrogen penetration freeze equipment.

altitude $807 \pm 4 \mathrm{~m}$ ) for normal rewarming. Blood circulation of frostbite tissue was examined and evaluated at 3-day intervals during postoperative wound healing. Vital signs and histologic determination of frostbite were observed 48 hours postoperatively.

\section{ESTIMATION OF VITAL SIGNS}

Vital signs reflect the vital movements and health of organisms. ${ }^{27}$ Therefore, we investigated the health situation of animals during the early stage of frostbite by measuring the following vital signs of the control, S-HAF, PT-HAF, and FT-HAF groups: systolic blood pressure (SBP), body temperature (BT), saturation of arterial blood oxygenation as measured by pulse oximetry $\left(\mathrm{SpO}_{2}\right)$, and respiratory rate (RR). The vital signs of each rat under sodium pentobarbital anesthesia $(65 \mathrm{mg} /$ $\mathrm{kg}$ body mass) were recorded using an electrophysiologic apparatus (MP150, BIOPAC Systems Inc, Goleta, CA) after 48 hours of frostbite. The SBP was observed using tail cuff blood pressure.

The metabolism of animals may be influenced by cold and hypoxia. Thus, $2 \mathrm{~mL}$ of arterial blood samples was collected from the abdominal aorta to estimate lipid peroxidation, which indirectly reflects the degree of cell injury in the metabolic process. ${ }^{28}$ Serum was obtained by centrifuging the samples at $4000 \mathrm{rpm}$ for 15 minutes at $4^{\circ} \mathrm{C}$ and stored at $-80^{\circ} \mathrm{C}$ for assessment. Lipid peroxidation was determined by estimating the level of thiobarbituric acid (TBA)-reactive substances via the Ohkawa method. ${ }^{29-31}$ Approximately $2.5 \mathrm{~mL}$ of $100 \mathrm{~g} / \mathrm{L}$ TBA solution was then homogenized with $0.5 \mathrm{~mL}$ of homogenate in each tube. The mixture was heated in a water bath at $95^{\circ} \mathrm{C}$ for 20 minutes and then cooled down with running water. The mixture was centrifuged at 2000 rpm for 10 minutes. An additional $2 \mathrm{~mL}$ of supernatant was added to $1 \mathrm{~mL}$ of the $6.7 \mathrm{~g} / \mathrm{L}$ TBA solution in a test tube. The solution was heated and cooled using the same method. Finally, the solution absorbance was measured at $532 \mathrm{~nm}$ by a spectrophotometer (ND-NDL-US-CAN, Thermo Fisher Scientific, Waltham, MA). Malondialdehyde (MDA) content was calculated using the MDA extinction coefficient $(155 / \mathrm{mM} \cdot \mathrm{cm}){ }^{32}$

\section{MICROCIRCULATION ASSESSMENT}

Determination of the blood circulation was important for evaluating injury and healing degree of a wound..$^{33,34}$ The blood circulation of the frostbite tissues in the different groups was imaged and compared using contrast-enhanced ultrasonography during wound healing, starting from the first postoperative day. The Sprague-Dawley rats were placed and fixed on an examination platform in the prone position to ensure that the rat frostbite areas were facing upward. Approximately $5 \mathrm{~mL}$ of $0.9 \%(\mathrm{w} / \mathrm{v})$ sodium chloride was placed into a small bottle of the contrast medium of SonoVue (SonoVue, Bracco Suisse SA, Manno, Switzerland) and shaken to obtain a microbubble suspension. The rats were anesthetized with sodium pentobarbital $(65 \mathrm{mg} / \mathrm{kg}$ body mass), and $0.1 \mathrm{~mL}$ of the microbubble suspension was injected into the tail vein at $1 \mathrm{~mL} / \mathrm{s}$. Then, $5 \mathrm{~mL}$ of the $0.9 \%(\mathrm{w} / \mathrm{v})$ sodium chloride was injected. A supersonic image from the first 5 seconds before the medium appearance to 50 seconds after medium injection was taken for dynamic analysis at offline state. Microbubble concentration (MC, $10^{9}$ [number/milliliter] $\mathrm{n} / \mathrm{mL}$ ) could quantitatively represent the status of the microcirculation, although microcirculation could also be observed and evaluated directly from the supersonic image. The size of $99 \%$ of supersonic microbubbles was less than 10 $\mu \mathrm{m}$. Thus, the microbubbles in the area of interest could be counted based on the representative supersonic image with Image Pro-Plus Version 4.5 for Windows software (Image Pro-Plus, Media Cybernetics, Inc, Rockville, MD). The MC per milliliter could be calculated and estimated. Finally, the MC was obtained to compare and analyze quantitatively the microcirculatory changes in the medium with different degrees of frostbite area. The $\mathrm{MC}$ in the different tissue layers among S-, PT-, and FTHAF groups was observed and compared. In addition, the MC of the PT-HAF group was compared with that of the PT-NF group.

\section{HISTOLOGIC OBSERVATIONS}

We observed the histologic changes and injury depth of HAF rats 48 hours postoperatively to investigate the pathohistologic process of HAF. Before sacrifice, photographs of the wounds in rats in the S-HAF, PT-HAF, 
Table. Vital signs of the four groups at $48 \mathrm{~h}$ after operation

\begin{tabular}{lccrc}
\hline Vital Signs & Control $(\mathrm{n}=8)$ & S- HAF $(\mathrm{n}=8)$ & PT- HAF $(\mathrm{n}=8)$ & FT- HAF $(\mathrm{n}=8)$ \\
\hline SBP (mm Hg) & $133 \pm 5$ & $117 \pm 4^{a}$ & $99 \pm 2^{a}$ & $85 \pm 3^{a}$ \\
$\mathrm{BT}\left({ }^{\circ} \mathrm{C}\right)$ & $37.6 \pm 0.5$ & $36.1 \pm 0.3^{a}$ & $35.2 \pm 0.5^{a}$ & $34.6 \pm 0.3^{a}$ \\
$\mathrm{Spo}_{2}(\%)$ & $91 \pm 4$ & $85 \pm 2^{a}$ & $79 \pm 4^{a}$ & $72 \pm 5^{a}$ \\
$\mathrm{RR}$ (breaths/min) & $71 \pm 5$ & $97 \pm 2^{a}$ & $132 \pm 3^{a}$ & $176 \pm 2^{a}$ \\
\hline
\end{tabular}

Values are expressed as mean $\pm \mathrm{SD}$.

${ }^{a} P<.05$ vs control group.

BT, body temperature; FT-HAF, full-thickness high altitude frostbite; PT-HAF, partial-thickness high altitude frostbite; RR, respiratory rate; SBP, systolic blood pressure; S-HAF, superficial high altitude frostbite; $\mathrm{Spo}_{2}$, arterial oxygen saturation by pulse oximetry.

FT-HAF, and PT-NF groups were obtained and compared. Then, after euthanizing the animals from each group, frostbite tissue samples were excised, immersed directly, and then fixed in $10 \%$ formalin for 60 hours. The tissue samples were embedded in paraffin wax after fixation. Consecutive 5 - $\mu \mathrm{m}$-thick sections were cut from each paraffin block with a saw microtome (Leica RM 2155, Leica Instruments, Nussloch, Germany). Tissue-containing slides were then deparaffinized, rehydrated, and stained with hematoxylin and eosin. Slides were observed under a light microscope. The severity and difference in histologic lesions among the S-HAF, PT-HAF, FT-HAF, and PT-NF groups were evaluated.

\section{STATISTICAL ANALYSIS}

All data in the present study are expressed as mean \pm SD. Data were analyzed using SPSS version 10.0 statistical software for Windows (SPSS, Chicago, IL). One-way analysis of variance was used to assess differences between the 5 groups and analyze the vital signs, microcirculation, and histologic changes. Once significant difference was detected, the significances between every 2 groups were determined using Fisher's least significant difference $t$ test. A probability value of less than .05 was considered statistically significant.

\section{Results \\ VITAL SIGNS}

The vital signs, namely $\mathrm{SBP}, \mathrm{BT}, \mathrm{Spo}_{2}$, and RR, of the 4 groups at 48 hours after operation are listed in the Table. The values of these vital signs, except for RR, in all HAF groups were lower than those of the control group. Moreover, the SBP, $\mathrm{BT}$, and $\mathrm{SpO}_{2}$ values significantly decreased with the frostbite degree. Significant differences in these variables were observed between the control group and the S-HAF, PT-HAF, and FT-HAF groups.

\section{MDA CONTENT}

MDA is a breakdown production of polyunsaturated fatty acids, which determines the extent of peroxidation reaction. $^{30,31}$ The MDA content in the rats is shown in Figure 2. The S-HAF, PT-HAF, and FT-HAF groups exhibited marked increases in MDA content, contrary to the rats in the control group. In addition, a significant difference was observed between the control and HAF groups $(P<.05)$.

\section{MICROCIRCULATION OF FROSTBITE TISSUE}

The MC can represent quantitatively the microcirculation changes of tissues. The microcirculation changes in the different tissue layers among the 5 groups during wound healing are shown in Figure 3. The MC values of the different groups obviously varied in the same tissue layer. Thus, MC values of the PT-HAF group were higher than those of the FT-HAF group but lower than those of the SHAF group in the progression of wound healing in different tissue layers. Additionally, the difference of MC was significant in control, S-HAF, PT-HAF, and FT-HAF groups. In the dermis layer, only MC of the S-HAF group recovered to the level of the control group on the 28th day. The MCs of the PT-HAF and FT-HAF groups exhibited

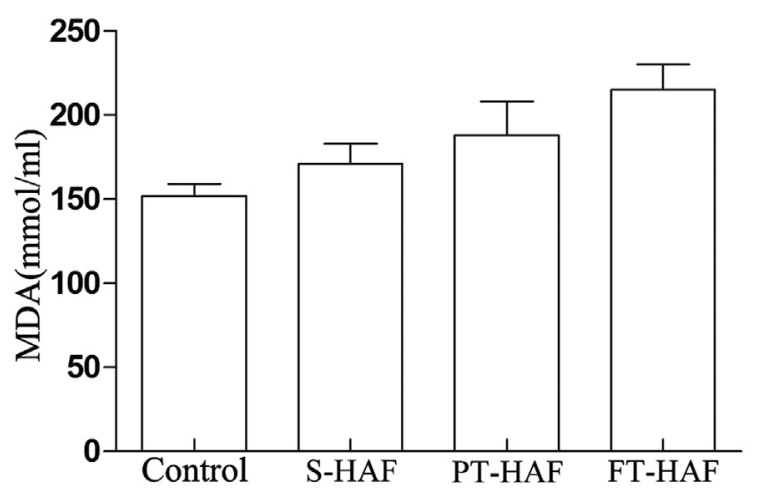

Figure 2. Changes in malondialdehyde (MDA) content in all groups. 

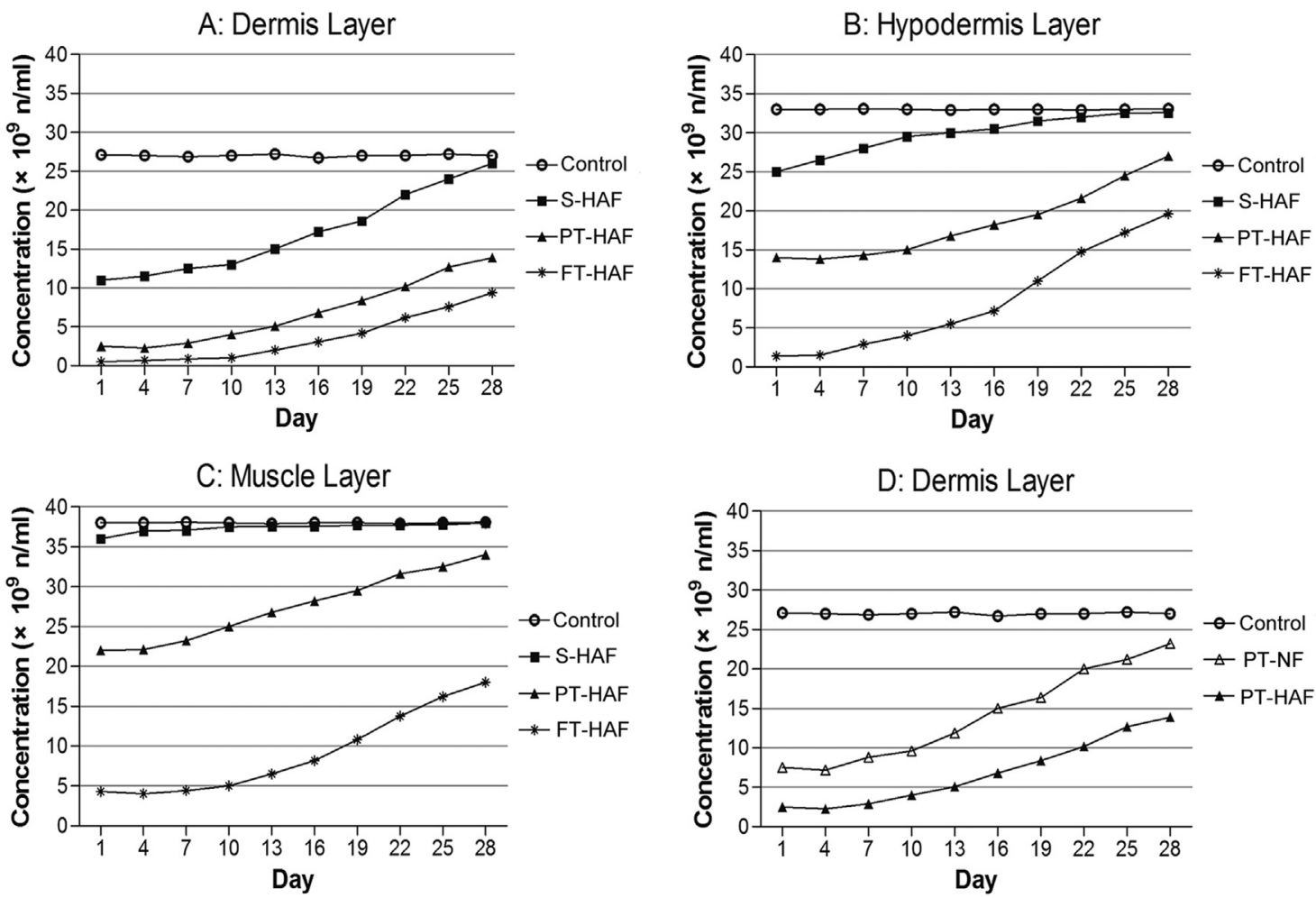

Figure 3. A, Comparison of microbubble concentration $(\mathrm{MC},(\mathrm{n} / \mathrm{mL}$, number/milliliter)) between control, superficial high altitude frostbite (S-HAF), partial-thickness high altitude frostbite (PT-HAF), and full-thickness high altitude frostbite (FT-HAF) groups in the dermis. B, C, MC comparison between control, S-HAF, PT-HAF, and FT-HAF groups in the hypodermis (B) and muscle layers (C). D, MC comparison between control, PT-HAF, and partial-thickness normal frostbite (PT-NF) groups in the dermis layer.

certain increases during wound healing, but the value of $\mathrm{MC}$ just reached approximately half of the MC the control group. Figure $3 \mathrm{~B}$ shows that the $\mathrm{MC}$ in the hypodermis layer was influenced greatly by frostbite in the FT-HAF group and almost reached zero. Figure $3 \mathrm{C}$ shows that only MC of the FT-HAF group was influenced greatly in the muscle layer. After 10 days, the MC of the FT-HAF group began to increase gradually. However, the value just reached $19.7 \pm$ $0.5 \times 10^{9} \mathrm{n} / \mathrm{mL}$ until the 28th day. Figure 3D illustrates that the MC of the PT-NF group was significantly higher than that of the PT-HAF group during wound healing in the dermis layer $(P<.05)$. Moreover, except for that of the SHAF and control groups, the MCs of groups decreased slightly within 48 hours, after which MC began to increase. This finding indicated that the injury of frostbite may increase slightly or remain constant.

\section{HISTOLOGIC STUDIES}

Macroscopic photographic and histologic differences of the frostbite tissues among the S-HAF, PT-HAF, FT-HAF, and PT-NF groups are shown in Figure 4. Figure 4A shows the macroscopic photograph indicating obvious blisters, erythema, and edema in the S-HAF group.
Figure 4B shows the appearance of HAF wound, some subcutaneous hemorrhage, and dead epidermis in the PTHAF group. Figure 4C shows the almost entirely dead epidermis and deeper tissue injury in the FT-HAF group. Figure 4D shows dead epidermis and a low incidence of subcutaneous hemorrhage in the PT-NF group. In addition, the injury in Figure 4D is obviously less than the injury in Figure 4B.

Detailed histologic changes are shown in Figures 4E through $4 \mathrm{H}$. Figure $4 \mathrm{E}$ shows that the S-HAF group exhibited a complete basic structure of the frostbite epidermis, local necrosis, hemorrhage foci, and infiltration of several types of inflammatory cells. A small focal amount of hemorrhage and edema could be observed in the superficial dermis layer. The deeper dermis and muscle layers were normal and did not show injured tissue. The fundamental pathologic changes of the PT-HAF group are shown in Figure 4F. The dermis layer demonstrated prominent edema, vascular dilatation and congestion, exudative hemorrhage, inflammatory cell infiltration, atrophy and necrotic hair follicle, and cracked collagen. Slight edema, inflammatory cell infiltration, and hemorrhage foci were observed in the muscle shadow layer. The deeper muscle layer was normal. 

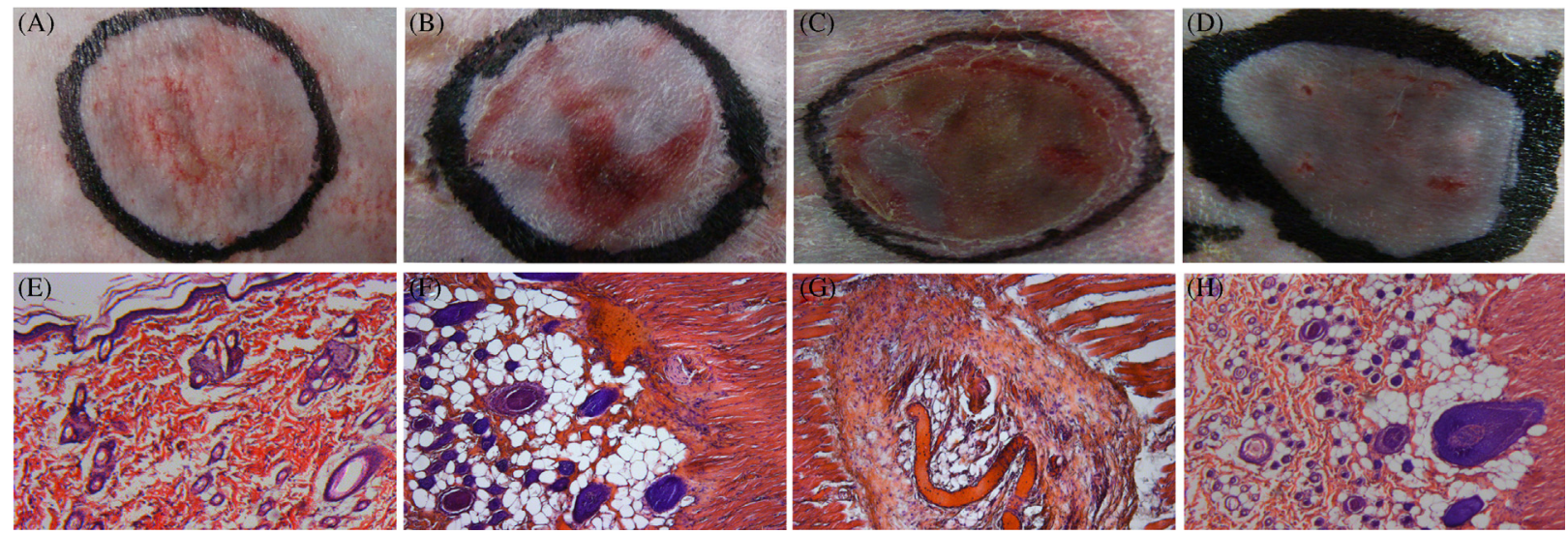

Figure 4. Macroscopic photographic and histologic examination of the frostbite tissues of superficial high altitude frostbite (S-HAF), partialthickness high altitude frostbite (PT-HAF), full-thickness high altitude frostbite (FT-HAF), and partial-thickness normal frostbite (PT-NF) groups. A, B, C, D, Macroscopic photographs of S-HAF (A), PT-HAF (B), FT-HAF (C), and PT-NF (D) groups. E, F, G, H, Histologic micrographs of S-HAF (E), PT-HAF (F), FT-HAF (G), and PT-NF (H) groups.

The epidermis and dermis layers exhibited necrosis, whereas the muscle layer was heavily damaged in the FT-HAF group (Figure 4G). The distance between the muscle fibers was increased. The muscle fiber interval was filled with tissue fluid. Additionally, the muscle layer demonstrated the denaturation of muscle bundles, vessel wall necrosis, stria disappearance of striated muscle, karyoplasm coagulation, a cell proliferationlike belt between muscular cells, and infiltration of many inflammatory cells. These results also further demonstrated that application of freezing duration for S-HAF, PT-HAF, and FT-HAF models is effective, and the HAF models were replicable. The fundamental pathologic changes in the PT-NF group are shown in Figure 4H. The superficial dermis demonstrated vascular dilatation and congestion, exudative hemorrhage, and inflammatory cell infiltration. By contrast, the deep partial part of the dermis remained nearly normal with a low degree of inflammatory cell infiltration.

\section{Discussion}

Pathophysiologic determination in different types of frostbite tissues is the prerequisite for screening optimal therapeutics. Cold and tissue hypoxia have been proven to be the main causes of frostbite injuries. ${ }^{23-26}$ However, the pathophysiologic changes of HAF caused by combined cold and hypoxia have not been described. Therefore, we improved the HAF models and evaluated the pathophysiologic process of HAF tissue under high altitude environment simulation in the current study.

Frostbite is normally categorized into 4 degrees according to the surface pathologic characteristics of wound, such as white plaque, erythema, blister, and necrosis. ${ }^{35}$ Furthermore, frostbite degrees were histologically classified by the absolute depth of frostbite injury of the dermis. ${ }^{17}$ However, the former method may be inaccurate, and the latter method is limited to the tissue layer of the dermis. In our SpragueDawley rat HAF models, we investigated the pathophysiology of the different tissue layers by defining the S-HAF, PT-HAF, and FT-HAF groups based on injury depth, thereby facilitating the accurate observation of the entire pathophysiology of HAF. Histologic results show that the S-HAF, PT-HAF, and FT-HAF models could be reproduced with freezing durations of 5,15 , and 28 seconds in high altitude simulation. According to histologic examination, the injuries of S-HAF only included the epidermis. The injury of PT-HAF extends from the epidermis to the reticular region of the dermis. The injury of FT-HAF reached the muscle layer. Moreover, the injury caused by HAF models was thicker than the injury caused by NF models, as shown by comparison of histologic changes between the PT-HAF and PT-NF groups. This phenomenon may have been caused by the fact that hypoxia caused increases in blood packed-cell volume, viscosity, and small vessel blockage, which accelerated the cold injury to form frostbite. Other reasons may include instruments and the operation of producing frostbite.

The results showed that the vital signs (ie, SBP, BT, and $\mathrm{SpO}_{2}$ ) of postoperative Sprague-Dawley rats decreased gradually with the frostbite severity, whereas RR significantly increased for cold injuries and hypoxia. MDA is a reliable marker of lipid peroxidation. ${ }^{29,30}$ MDA contents of the S-HAF, PT-HAF, and FT-HAF groups significantly increased, contrary to those of the control group. Although the damage to the metabolism of Sprague-Dawley rats resulting from cold and hypoxia could not be observed directly, the change in MDA 
could indicate that the metabolism of Sprague-Dawley rats had been influenced by HAF. ${ }^{28}$ The detected vital signs also showed that the change in MDA and the values of $\mathrm{SBP}, \mathrm{BT}$, and $\mathrm{Spo}_{2}$ may be highly correlated. In other words, changes in SBP, BT, and $\mathrm{SpO}_{2}$ could be the external representation of tissue metabolic injury, which still needs further in-depth study. The vital signs of animals were influenced significantly by the cold and hypoxia injuries, but no unexpected deaths occurred in the frostbite method during operation.

Changes in the microcirculation and histology of frostbite tissues were closely associated with the severity degree of frostbite. Contrast-enhanced ultrasonography images and $\mathrm{MC}$ indicate that the different durations of freezing combined with tissue hypoxia exerted different influences on the blood circulation of frostbite tissues. In particular, evident differences were observed among the control, S-HAF, PT-HAF, and FT-HAF groups in the different tissue layers. Cold combined with tissue hypoxia caused more damage to the microcirculation of frostbite tissue than that of cold only. Healing progression of the HAF group was significantly slower than that of the NF group, probably because HAF causes more injury to the blood circulation than NF. As expected, histologic observation proved that the destruction to the S-HAF, PT-HAF, and FT-HAF groups had extended from the epidermis to the muscle layers with degree of the frostbite. Damages in the $3 \mathrm{HAF}$ groups varied, which further indicates that the results of histologic observation were coincident with the results of the MC method. Therefore, the MC method for evaluating the tissue microcirculation could effectively distinguish the injury layer and depth of frostbite. The method has powerful operability and practicability. Thus, the MC method has the potential for distinguishing the layers between healthy and damaged tissues, which could be applied to excision operations of burn or frostbite.

In our study, 48 hours after operation was chosen to obtain vital signs and histologic determination for the following reasons. At the early stage of frostbite injury, the ice crystals caused direct mechanical damage to cells. ${ }^{36}$ Then, thrombosis caused by the Hunting reaction and vasoconstriction causes more injury to the tissue. ${ }^{37}$ Furthermore, activation of inflammatory cells causes damage to the circulation. ${ }^{38}$ The circulatory recovery also proved the progression. Thus, the frostbite tissue was assumed to exhibit physical and chemical changes because of the injury from cold and hypoxia at 48 hours postoperatively. The pathophysiologic changes became obvious and observable at that time. In addition, granulation tissue was not discernible from the histologic observation at 48 hours postoperatively. Hence, we choose this period to evaluate the pathophysiologic changes of HAF.

\section{LIMITATIONS}

Several limitations of our research were identified through the experiments. Because the high altitude simulation environment of constant cold, low pressure, and hypoxia may cause too-low rat survival rates, the rats were frozen by liquid nitrogen in the large hypobaric chamber to simulate accidental HAF. These models could not completely simulate all kinds of ideal HAF. The occurrence of human HAF requires temperatures higher than $-196^{\circ} \mathrm{C}$ and longer freezing times. $^{6}$ Therefore, in our future work, better HAF models will be created by redesigning the hypobaric chamber and the temperature control of the rats, which could ensure the survival rates of rats during the construction of HAF models. Furthermore, we could find in the macroscopic photographs that the injury of the rounded wound was not uniform, probably because the dorsum of rats is not smooth, in contrast to the smooth aluminum alloy bottom. Thus, the main differences between the S-HAF, PT-HAF, and FTHAF groups were distinguished based on the injury depth of frostbite rather than the amount of dead tissue in a certain area. Although we focused on the worst part of the frostbite wound, a more suitable freezing instrument to match different parts of the rat body should be designed to generate a more uniform freezing injury. Moreover, MC detection, which was obtained from the estimation of the count of the supersonic image, was not precisely accurate. However, the MC could represent the situation of the microcirculation in the study. Finally, histologic differences between the HAF and NF groups were difficult to observe by quantitative methods, so a suitable method to compare the histologic differences between HAF and NF will be explored in our future work.

\section{Conclusions}

The improved HAF models show that changes in the pathophysiology of the different degrees of HAF caused by cold and hypoxia were observed and obtained. HAF caused more damage to the microcirculation than NF. The pathophysiology of HAF was specific and obviously varied with the degree of frostbite injuries. Consequently, the pathophysiologic determination of HAF will provide a powerful and important support for studying frostbite treatment. 
Acknowledgments: The authors sincerely thank Prof Xiaodong Zhou, Zhongming Wang, and Dr Yong Fu for their technical help in the experiments.

Author Contributions: Study concept and design (JH, HL, MJ); obtaining funding (MJ); acquisition of the data (JH, HL, XG, LJ, LL); analysis of the data (HL, XG, LJ, HS, MJ); drafting of the manuscript (JH, HS); critical revision of the manuscript (JH, MJ); and approval of final manuscript (MJ).

Financial/Material Support: This work was supported by the National Natural Science Foundation of China (Grant No. 81301344). Disclosures: None.

\section{References}

1. Britt LD, Dascombe WH, Rodriguez A. New horizons in management of hypothermia and frostbite injury. Surg Clin North Am. 1991;71:345-370.

2. Knize DM, Weatherley-White RC, Paton BC, Owens JC. Prognostic factors in the management of frostbite. $J$ Trauma. 1969;9:749-759.

3. Murphy JV, Banwell PE, Roberts AH, McGrouther DA. Frostbite: pathogenesis and treatment. J Trauma. 2000;48: 171-178.

4. Ervasti O, Juopperi K, Kettunen P, et al. The occurrence of frostbite and its risk factors in young men. Int $J$ Circumpolar Health. 2004;63:71-80.

5. Moore GW, Semple JL. Freezing and frostbite on Mount Everest: new insights into wind chill and freezing times at extreme altitude. High Alt Med Biol. 2011;12:271-275.

6. Harirchi I, Arvin A, Vash JH, Zafarmand V. Frostbite: incidence and predisposing factors in mountaineers. $\mathrm{Br} \mathrm{J}$ Sports Med. 2005;39:898-901.

7. Keskin M, Tosun Z, Duymaz A, Savaci N. Frostbite injury due to improper usage of an ice pack. Ann Plast Surg. 2005;55:437-438.

8. Golant A, Nord RM, Paksima N, Posner MA. Cold exposure injuries to the extremities. J Am Acad Orthop Surg. 2008;16:704-715.

9. Ikäheimo TM, Hassi J. Frostbites in circumpolar areas. Glob Health Action. 2011;4:8456-8463.

10. McIntosh SE, Hamonko M, Freer L, et al. Wilderness Medical Society. Wilderness Medical Society practice guidelines for the prevention and treatment of frostbite. Wilderness Environ Med. 2011;22:156-166.

11. Breetveld M, Richters CD, Rustemeyer T, Scheper RJ, Gibbs S. Comparison of wound closure after burn and cold injury in human skin equivalents. J Invest Dermatol. 2006;126:1918-1921.

12. Bruen KJ, Ballard JR, Morris SE, Cochran A, Edelman LS, Saffle JR. Reduction of the incidence of amputation in frostbite injury with thrombolytic therapy. Arch Surg. 2007; 142:546-553.

13. Jurkovich GJ. Environmental cold-induced injury. Surg Clin North Am. 2007;87:247-267.

14. Folio LR, Arkin K, Butler WP. Frostbite in a mountain climber treated with hyperbaric oxygen: case report. Mil Med. 2007;172:560-563.
15. Zook N, Hussmann J, Brown R, et al. Microcirculatory studies of frostbite injury. Ann Plast Surg. 1998;40: 246-255.

16. Twomey JA, Peltier GL, Zera RT. An open-label study to evaluate the safety and efficacy of tissue plasminogen activator in treatment of severe frostbite. J Trauma. 2005;59:1350-1354.

17. Rothenberger J, Held M, Jaminet P, et al. Development of an animal frostbite injury model using the GoettingenMinipig. Burns. 2014;40:268-273.

18. Auerbach LJ, Galvez MG, De Clerck BK, et al. A novel mouse model for frostbite injury. Wilderness Environ Med. 2013;24:94-104.

19. Auerbach LJ, DeClerk BK, Fathman CG, Gurtner GC, Auerbach PS. Poly-L-arginine topical lotion tested in a mouse model for frostbite injury. Wilderness Environ Med. 2014;25:160-165.

20. Goertz O, Baerreiter S, Ring A, et al. Determination of microcirculatory changes and angiogenesis in a model of frostbite injury in vivo. J Surg Res. 2011;168:155-161.

21. Goertz O, Hirsch T, Buschhaus B, et al. Intravital pathophysiologic comparison of frostbite and burn injury in a murine model. J Surg Res. 2011;167:e395-e401.

22. Sarikaya I, Aygit AC, Candan L, Sarikaya A, Türkyilmaz M, Berkarda S. Assessment of tissue viability after frostbite injury by technetium-99m-sestamibi scintigraphy in an experimental rabbit model. Eur $J$ Nucl Med. 2000;27:41-45.

23. Boutin AT, Weidemann A, Fu Z, et al. Epidermal sensing of oxygen is essential for systemic hypoxic response. Cell. 2008; 133:223-234.

24. Windberger U, Spurny K, Graf A, Thomae H. Hemorheology in experimental research: is it necessary to consider blood fluidity differences in the laboratory rat? Lab Anim. 2015;49:142-152.

25. Durand J, Varene P, Jacquemin C. Cardiac output and regional blood flows in altitude residents. In: Brendel W, Zink RA, eds. High Altitude Physiology and Medicine. New York, NY: Springer-Verlag; 1982:129-141.

26. Ay H, Yildiz S, Uzun G, Solmazgul E, Dundar K, Yildirim I. The treatment of deep frostbite with hyperbaric oxygen. Inj Extra. 2005;36:499-502.

27. Matsuda A, Kimura M, Itokawa Y. Influence of selenium deficiency on vital functions in rats. Biol Trace Elem Res. 1998;61:287-301.

28. De Ita JR, Castilla-Cortázar I, Aguirre GA, et al. Altered liver expression of genes involved in lipid and glucose metabolism in mice with partial IGF-1 deficiency: an experimental approach to metabolic syndrome. J Transl Med. 2015;13:326.

29. Ohkawa H, Ohishi N, Yagi K. Assay for lipid peroxides in animal tissues by thiobarbituric acid reaction. Anal Biochem. 1979;95:351-358.

30. Koca K, Yurttas Y, Bilgic S, et al. Effect of preconditioned hyperbaric oxygen and ozone on ischemiareperfusion induced tourniquet in skeletal bone of rats. J Surg Res. 2010;164:e83-e89. 
31. Gu C, Li S, Zhao L, Song X, Qin G. The effects of soybean trypsin inhibitor on free radicals levels in pancreatic mitochondria of mice. J Food Nutr Res. 2014;2:357-362.

32. Fu J, Huang B. Involvement of antioxidants and lipid peroxidation in the adaptation of two cool-season grasses to localized drought stress. Environ Exp Bot. 2001;45: 105-114.

33. Kisch T, Sorg H, Forstmeier V, et al. Remote effects of extracorporeal shock wave therapy on cutaneous microcirculation. J Tissue Viability. 2015;24:140-145.

34. Yang Y, Wang F, Yin D, Fang Z, Huang L. Astragulus polysaccharide-loaded fibrous mats promote the restoration of microcirculation in/around skin wounds to accelerate wound healing in a diabetic rat model. Colloids Surf B Biointerfaces. 2015;136:111-118.

35. Reamy BV. Frostbite: review and current concepts. J Am Board Fam Pract. 1998;11:34-40.

36. Bracker MD. Environmental and thermal injury. Clin Sports Med. 1992;11:419-436.

37. McCauley RL, Heggers JP, Robson MC. Frostbite. Methods to minimize tissue loss. Postgrad Med. 1990;88(67-68):73-77.

38. Robson MC, Heggers JP. Evaluation of hand frostbite blister fluid as a clue to pathogenesis. J Hand Surg Am. 1981;6: 43-47. 